\title{
Erratum to \\ Hallgren, K. A. (2012). Computing inter-rater reliability for observational data: An overview and tutorial.
}

\begin{abstract}
An erratum was found in Hallgren, K. A. (2012). Computing inter-rater reliability for observational data: An overview and tutorial. The error and correction are presented here.
\end{abstract}

The text on page 29 of the original article and in Tables 4 and 7 state that SPSS computes the Siegel and Castellan (1988) variant of Cohen's kappa. This is incorrect. When SPSS output was compared against various computation methods, SPSS (version 17) computed Cohen's kappa according to Cohen's (1960) original method. This finding also conforms to the algorithm for computing kappa reported by SPSS (SPSS Inc., 2007, p. 123).

Additionally, the $\mathrm{R}$ concord package appears to incorrectly compute the Cohen's kappa statistic, and this package is no longer being maintained and was suggested for removal from the Comprehensive $\mathrm{R}$ Archive Network (CRAN). Thus, it is recommended that Cohen's kappa be estimated via alternative methods, such as the kappa2 function of the irr package (Gamer, Lemon, Fellows, \& Sing, 2010).

\section{References}

Gamer, M., Lemon, J., Fellows, I., \& Sing, P. (2010). irr: Various coefficients of interrater reliability and agreement (Version 0.83) [software]. Available from http://CRAN.Rproject.org/package=irr.

Hallgren, K. A. (2012). Computing inter-rater reliability for observational data: An overview and tutorial. Tutorials in Quantitative Methods for Psychology, 8, 23-34.

SPSS Inc. (2007). SPSS Statistics 17.0: Algorithms. Chicago, IL: SPSS Inc. 